\title{
Reflets
}

Revue ontaroise d'intervention sociale et communautaire

\section{Le travail obligatoire en Ontario solution ou imposture?}

\section{Chantal Cholette}

Volume 4, numéro 1, printemps 1998

Intervention en contextes minoritaires

URI : https://id.erudit.org/iderudit/026199ar

DOI : https://doi.org/10.7202/026199ar

Aller au sommaire du numéro

Éditeur(s)

Reflets : Revue ontaroise d'intervention sociale et communautaire

ISSN

1203-4576 (imprimé)

1712-8498 (numérique)

Découvrir la revue

Citer cet article

Cholette, C. (1998). Le travail obligatoire en Ontario solution ou imposture? Reflets, 4(1), 100-126. https://doi.org/10.7202/026199ar

\section{Résumé de l'article}

Tel que promis au printemps 1995 lors de sa campagne électorale, le gouvernement de MikeHarris déposait le 12 juin 1997 un projet de loi visant à réformer radicalement le système del'aide sociale en Ontario. Le programme de travail obligatoire Ontario au travail est un deséléments clés de cette réforme. Toutefois, plutôt que d'offrir de véritables solutions au chômageendémique, ce programme met fin à d'importants acquis sociaux. Que se cache-t-il derrière lediscours du gouvernement Harris... réforme judicieuse ou paroles fielleuses à l'égard des plusdémunis de notre société?
Tous droits réservés (C) Reflets : Revue ontaroise d'intervention sociale et communautaire, 1998
Ce document est protégé par la loi sur le droit d'auteur. L'utilisation des services d’Érudit (y compris la reproduction) est assujettie à sa politique d'utilisation que vous pouvez consulter en ligne.

https://apropos.erudit.org/fr/usagers/politique-dutilisation/ 


\section{Le travail obligatoire en Ontario : solution ou imposture? ${ }^{1}$}

Tel que promis au printemps 1995 lors de sa campagne électorale, le gouvernement de Mike Harris déposait le 12 juin 1997 un projet de loi visant à réformer radicalement le système de l'aide sociale en Ontario. Le programme de travail obligatoire Ontario au travail est un des éléments clés de cette réforme. Toutefois, plutôt que d'offrir de véritables solutions au chômage endémique, ce programme met fin à d'importants acquis sociaux. Que se cache-t-il derrière le discours du gouvernement Harris... réforme judicieuse ou paroles fielleuses à l'égard des plus démunis de notre société?

\section{Chantal Cholette ${ }^{2}$}

La coopérative Convergence, groupe membre de la Table féministe francophone de concertation provinciale de l'Ontario

\section{Introduction}

Dans cet article, nous présenterons rapidement quelques faits saillants de la réforme de l'aide sociale en Ontario ainsi qu'une description sommaire du programme Ontario au travail. Nous décrirons également les contextes politique et social dans lesquels s'insère la venue d'un programme de travail obligatoire en Ontario, ainsi que l'historique de ce type de programmes.

Notre but consiste à préciser certains enjeux du programme de travail obligatoire Ontario au travail, un des deux volets de la réforme sociale proposée par le gouvernement Harris. À chacun des objectifs énoncés dans la réforme, nous démontrerons l'écart qu'il y a entre le discours (la carotte) et la réalité (le bâton). Avant de conclure, 
«Notre objectif est de contribuer à la déconstruction de mythes en présentant des renseignements factuels pour faire contrepoids aux demi-vérités qu'affirment les personnes politiques quand elles parlent $d u$ système de l'aide sociale en Ontario et de ses bénéficiaires.» nous étudions l'impact de la mise en oeuvre du programme Ontario au travail sur les femmes chefs de famille monoparentale.

Notre objectif est de contribuer à la déconstruction de mythes en présentant des renseignements factuels pour faire contrepoids aux demi-vérités qu'affirment les personnes politiques quand elles parlent du système de l'aide sociale en Ontario et de ses bénéficiaires. Nous incitons également les lectrices et lecteurs à explorer ce qui se cache derrière l'apparence de franchise que fait miroiter le gouvernement Harris quand il proclame que le nouveau système d'aide sociale, tel qu'il a l'intention de le modifier, «aurait pour but l'autonomie, (...) qu'il donnerait aux gens les possibilités de devenir maitres de leur vie» (communiqué :l'Ontario tient sa promesse...).

Enfin, cet article se veut un essai, c'est-à-dire que nous ne prétendons pas épuiser le sujet. Notre démarche s'inscrit plutôt dans un processus de questionnement continu qui consiste à comprendre les fondements du programme de travail obligatoire ontarien, à jeter la lumière sur les nombreuses contradictions que nous avons pu relever au cours de la dernière année.

\section{Le contexte ontarien : un raz de maré néolibéral sans précédent}

Sous la bannière de la "Révolution du bon sens», la campagne électorale du Parti conservateur de l'Ontario a produit des résultats surprenants. Si plusieurs commentateurs et analystes politiques avaient prévu que les néodémocrates ne seraient probablement pas reportés au pouvoir, peu d'entre eux avaient envisagé la possibilité d'une majorité écrasante des Conservateurs. Depuis, les petites et grandes communautés ontariennes sont estomaquées par l'ampleur d'un véritable raz de marée néolibéral. Plusieurs intervenantes et intervenants s'inquiètent des conséquences à moyen et long termes des nombreuses politiques néolibérales sur les acquis sociaux et juridiques durement gagnés depuis la deuxième guerre mondiale. Comme l'écrivait Malraux, «on craint 
ces raz de marée qui emportent, avec les valeurs d'une société, notre société elle-même».

Rappelons que la «Révolution du bon sens» reposait sur cinq éléments clés: diminuer de $30 \%$ le taux d'impôt provincial sur le revenu, diminuer les dépenses publiques de $20 \%$, supprimer les obstacles à la croissance, mieux réussir à moindre coût et équilibrer le budget de la province. De plus, les Conservateurs de Mike Harris promettaient de créer plus de 725000 nouveaux emplois au cours de leur premier mandat.

Dès son arrivée au pouvoir en juin 1995 et fort de son imposante victoire, le gouvernement Harris donnait le ton des nombreuses réformes qu'il avait l'intention de mettre en oeuvre. Le 9 juin 1995, au lendemain de l'élection, Mike Harris abolit la Loi sur l'équité en matière d'emploi. La population ontarienne devait immédiatement comprendre que le gouvernement avait l'intention de tenir ses promesses et qu'il n'arrêterait devant rien ni personne pour rencontrer ses objectifs. Quatre mois plus tard, il s'en prend au système de l'aide sociale.

À la lumière des politiques mises en oeuvre par le gouvernement de l'Ontario depuis son élection en juin 1995, en particulier celles annoncées dans le cadre de la "méga-semaine» ${ }^{3}$ et des recommandations de la Commission de révision des formalités administratives (RedTape Commission) ${ }^{4}$, il est évident que la priorité du gouvernement Harris est de promouvoir les intérêts de la classe d'affaires et ceux de la finance.

Le gouvernement ne semble retenir qu'un seul critère dans sa «révolution du bon sens»: créer des conditions optimales pour une exploitation maximale des travailleuses et des travailleurs par le secteur privé. Dans la plus pure tradition néolibérale, il est en train de forcer la société ontarienne à retourner à un mode de production où ce sont les «forces du marché» qui vont dicter le développement économique, social, sanitaire et environnemental de la province. De la sorte, le gouvernement est en train d'abdiquer sa fonction de redistribution économique et de protection des droits sociaux et des autres droits fondamentaux de la population. 
Au cours des six premiers mois de son mandat, le gouvernement Harris a annoncé d'importantes réductions dans les budgets réservés aux services sociaux et de la santé (par exemple, des réductions de 1,3 milliards dans les services hospitaliers et de 800 millions de dollars dans l'éducation). De plus, le gouvernement Harris a non seulement éliminé plusieurs programmes dont le fonds de soutien aux nouvelles garderies à but non lucratif et le financement destiné à la prévention de la violence conjugale, mais il a également aboli certaines lois (comme la Loi 40 qui interdisait notamment le recours aux briseurs de grève) de même que la gratuité des médicaments pour 380000 personnes âgées et 1,3 millions de bénéficiaires de l'aide sociale en imposant un ticket modérateur de $2 \$$ pour chaque achat de médicament.

Enfin, la dévolution d'une foule de programmes et de services sociaux et communautaires aux municipalités (et par la force des choses, au secteur privé) aura de nombreuses conséquences. La décentralisation des programmes sociaux risque de créer des villes

"C'est donc dans un climat d'austérité économique et de réduction sinon d'élimination d'importants acquis sociaux que s'insère le programme de travail obligatoire Ontario au travail.» fermées qui imposent des contraintes incroyables visant à limiter l'accès aux programmes et services d'aide sociale, restreignant ainsi la mobilité des citoyens et des citoyennes. Puisque le gouvernement impose une interdiction aux municipalités de faire des déficits, il nous semble évident que ces dernières tenteront, par tous les moyens, de limiter leurs dépenses sociales et d'accroître leurs revenus en augmentant les impôts fonciers.

C'est donc dans un climat d'austérité économique et de réduction sinon d'élimination d'importants acquis sociaux que s'insère le programme de travail obligatoire Ontario au travail.

\section{Le travail obligatoire : éclair de génie ou plat réchauffé?}

\section{Un bref rappel historique ${ }^{5}$}

Nous croyons qu'il est important de connaitre son histoire, d'en tirer des leçons et surtout, d'éviter de répéter perpétuellement les mêmes erreurs. C'est justement pour contribuer au façonnage de 
"... le lien entre le travail obligatoire et l'aide sociale est probablement au centre d'un des plus anciens débats sociaux de l'histoire." la mémoire collective que nous avons retracé les événements historiques suivants.

Le parti Conservateur de l'Ontario n'a pas fait preuve d'originalité en incluant le concept du "workfare» dans sa «révolution du bon sens». En effet, le lien entre le travail obligatoire et l'aide sociale est probablement au centre d'un des plus anciens débats sociaux de l'histoire. Au $16^{\mathrm{e}}$ siècle, alors que l'Angleterre adoptait ses premières «Poor Laws», la croyance populaire voulait que «tous ceux que Dieu, et non leur paresse, a rendu pauvres, doivent être aidés» ${ }^{6}$. Il y a donc longtemps que certaines personnes divisent les pauvres en deux clans distincts :les premiers «méritent» l'aide sociale et les deuxièmes en sont indignes. Ce genre de discours lie l'Angleterre de l'époque élisabéthaine à l'Ontario de Mike Harris.

Une des plus récentes initiatives canadiennes de travail obligatoire remonte à l'époque de la Grande dépression. À l'été de 1933, alors que plus de 400000 Ontariens étaient sans emploi, le gouvernement fédéral de R.B. Bennett établissait un réseau de camps de travail partout au Canada, supervisés par le ministère de la Défense nationale. Tous les hommes sans emploi et célibataires âgés de 16 ans et plus devaient s'y "enrôler» pour recevoir de l'aide sociale. L'objectif principal de ces camps était d'imposer des travaux aux chômeurs et non de leur fournir des emplois. En retour de leurs labeurs, les hommes étaient nourris et hébergés mais ne recevaient pas de salaire. À sa place, on leur accordait une maigre allocation de 0,20 \$ par jour pour leurs dépenses personnelles (Struthers 1996:1-3).

Suite à l'élection du Libéral Mitch Hepburn à l'été de 1934, l'Ontario mettait en oeuvre son programme de "work tests» auxquels étaient tenus de participer le reste des sans-emploi, c'està-dire les hommes mariés et chefs de famille. Peu de temps après leur élection, Mitch Hepburn et David Croll (alors ministre du Bien-être social de l'Ontario) apprenaient du gouvernement Bennett que le gouvernement fédéral allait couper ses subventions d'aide sociale à la province de $37 \%$. En imposant cette coupe, Ottawa cherchait désespérément à équilibrer son budget.

Un mois après avoir été porté au pouvoir, Croll annonçait à l'Assemblée législative que l'escroquerie sévissait dans les 
«En 1934, tous les bénéficiaires ont été obligés de placer une nouvelle demande d'aide sociale et de déclarer sous serment qu'ils étaient vraiment pauvres, sans ressources. On a créé des escouades anti-fraude qui faisaient des visites surprises au domicile des bénéficiaires pour découvrir des sources de revenu non déclarées.» programmes d'aide sociale. Il se disait également de plus en plus inquiet que des hommes aptes au travail bénéficient de denrées, d'un abri, de vêtements et d'autres produits sans qu'on ne leur demande rien en retour. Sous la devise «De l'aide pour les travailleurs, rien pour les paresseux» (Struthers 1993:91) ${ }^{7}$, Croll menait une lutte contre le spectre de la fraude sociale en employant des méthodes qui nous sont familières. En 1934, tous les bénéficiaires ont été obligés de placer une nouvelle demande d'aide sociale et de déclarer sous serment qu'ils étaient vraiment pauvres, sans ressources. On a créé des escouades anti-fraude qui faisaient des visites surprises au domicile des bénéficiaires pour découvrir des sources de revenu non déclarées.

La même année, Queen's Park diminuait les prestations d'aide sociale de $15 \%$ dans 40 villes. Croll imposait également les «work tests» aux chômeurs.Au cours de l'hiver 1934-35, le gouvernement ontarien informait les municipalités que les sans-emploi devaient être obligés de travailler en contrepartie de leurs prestations d'aide sociale. Les chômeurs pourraient regagner leurs pertes de prestations en travaillant un ou deux jours par semaine à scier du bois dans la cour municipale, à balayer les rues, à râteler les feuilles, à arracher les pissenlits, à pelleter de la neige ou à enlever les broussailles des terrains vacants. Ceux qui refusaient de travailler se voyaient tout simplement privés de toute aide sociale.

Au cours de l'hiver et de l'été de 1935, les bénéficiaires de l'aide sociale ont tenu des manifestations dans les comtés de Markham, Crowland, Windsor et London. Les manifestants demandaient l'une de deux choses : qu'on les paie mieux ou qu'on abolisse le programme de travail obligatoire. Dans le comté d'East York, 2700 familles ont participé à une manifestation dénonçant le travail obligatoire et les réductions dans les prestations de l'aide sociale. Lorsque les conseillers ont refusé de discuter ces demandes, les manifestants ont occupé la salle du conseil. La situation a dégénéré en émeute lorsque plus de 40 agents de la police provinciale ont été dépêchés sur les lieux. Il y avait, à cette période, des manifestations partout en province et on organisait des marches de la faim à Queen's Park. 
«En 1959, plusieurs municipalités de l'Ontario avaient commencé à rétablir les «work tests". À Chippewa (près de Niagara Falls), les femmes bénéficiaires de l'aide sociale devaient laver les fenêtres et nettoyer les planchers de l'hôtel de ville à raison de 16 heures par mois."
En 1941, la fin de la dépression marque aussi la fin des «work tests» ${ }^{8}$. C'est au cours de cette année-là qu'Ottawa, les provinces et les municipalités s'entendent à l'effet que toutes les personnes aptes au travail ne seront plus admissibles à l'aide sociale. Il est important ici de souligner que deux éléments clés ont facilité la venue de ce genre de politique.

D'abord, l'entrée en vigueur du programme national d'assurance chômage en 1940 a permis de répondre aux besoins d'une grande proportion de sans-emploi. Ensuite, l'extraordinaire prospérité qu'a connue l'Ontario (et l'ensemble du Canada) au cours des années 1940 et 1950 a fait en sorte que l'aide sociale n'était pratiquement plus nécessaire. Le taux de chômage à l'époque ne s'élevait qu'à un maigre $3 \%$.

Mais peu de temps après l'adoption de la Loi sur l'assurance chômage de $1956^{9}$, le taux de chômage s'est remis à grimper. Atteignant le seuil des $7 \%$ de chômage, les provinces ont connu une augmentation de $150 \%$ des cas d'aide sociale. En 1959, plusieurs municipalités de l'Ontario avaient commencé à rétablir les «work tests». À Chippewa (près de Niagara Falls), les femmes bénéficiaires de l'aide sociale devaient laver les fenêtres et nettoyer les planchers de l'hôtel de ville à raison de 16 heures par mois. Scarborough faisait travailler ses bénéficiaires des journées de huit heures à nettoyer les broussailles de terrains vacants.

En 1961, le vérificateur général du Canada étudiait le programme de travail obligatoire de l'Ontario. Après avoir découvert que la province utilisait les fonds d'aide au chômage pour subventionner des programmes de travail obligatoire, le ministère de la Santé et du Bien-être a avisé le Premier ministre John Robarts que les prestations ne devaient plus être distribuées par l'entremise de tels programmes.

Lorsque le Régime d'assurance publique du Canada $(R A P C)^{10}$ est entré en vigueur en 1966, il devenait dorénavant légalement impossible d'instituer des programmes de travail obligatoire. Une des clauses de ce programme stipulait que le droit de recevoir de l'aide sociale dépendait sur le fait, et non la cause, du besoin. En d'autres mots, toute personne dans le besoin, quelle qu'en soit la raison, avait le droit de bénéficier de l'aide sociale. Cette clause 
"Ce qu'il est intéressant de noter, c'est qu'à chacune des époques où les gouvernements ont imposé des programmes de travail obligatoire, les sociétés occidentales traversaient d'importantes périodes de transformation." précisait que, pour recevoir les paiements de péréquation du gouvernement fédéral, les provinces et les municipalités ne pouvaient pas exiger des bénéficiaires de travailler afin de toucher leurs prestations.

Malheureusement, le RAPC a été aboli par le gouvernement fédéral et remplacé en avril 1996 par le Transfert canadien en matière de santé et de programmes sociau $x^{11}$. Ce nouveau programme permet aux projets de travail obligatoire de refaire surface.

Ce qu'il est intéressant de noter, c'est qu'à chacune des époques où les gouvernements ont imposé des programmes de travail obligatoire, les sociétés occidentales traversaient d'importantes périodes de transformation. Les «Poor Laws» du $16^{\mathrm{e}}$ siècle sont apparues en Angleterre avec la révolution industrielle. Les camps de travail et les "work tests» prenaient naissance au Canada et en Ontario pendant les années de la Grande dépression et de la deuxième guerre mondiale. La deuxième vague des "work tests» refait surface en Ontario alors que des changements technologiques commençaient à bouleverser le monde du travail. Le programme Ontario au travail ou le "workfare» de Mike Harris apparaît au moment où le taux de chômage frise les $10 \%$, où plus de 1,1 millions d'Ontariennes et d'Ontariens dépendent de l'aide sociale pour survivre et où l'informatique et la robotique transforment radicalement le marché du travail, faisant disparaître les emplois comme neige au soleil.

À défaut de trouver de véritables solutions aux maux du moment, les gouvernements créent une diversion en accusant et en traquant les personnes les plus démunies de la société. En 1998, la société ontarienne saura-t-elle éviter de répéter les erreurs du passé? ${ }^{12}$

\section{Le programme Ontario au travail}

\section{$Q u^{\prime} e s t$-ce que le programme Ontario au travail?}

Selon un communiqué de presse diffusé en juin 1997 par le ministère des Services sociaux et communautaires (MSSC 1997a:2), 
le programme Ontario au travail «permet au système d'aide sociale de retrouver sa première vocation, celle d'un programme transitoire de dernier recours qui fournit aux bénéficiaires de l'aide sociale un tremplin pour réintégrer la population active». Cependant, et contrairement à cet énoncé, l'histoire démontre que la toute première vocation du système d'aide sociale était plutôt de venir en aide aux citoyennes et citoyens démunis afin de palier à leurs besoins essentiels.

«Selon le programme Ontario au travail... les bénéficiaires devront d'abord participer à un placement dans la collectivité ou suivre un programme de formation menant à un emploi pour avoir droit à leurs prestations d'aide sociale."
Selon le programme Ontario au travail (MSSC s.d.), les bénéficiaires devront d'abord participer à un placement dans la collectivité ou suivre un programme de formation menant à un emploi pour avoir droit à leurs prestations d'aide sociale. Seules les personnes âgées et les personnes handicapées sont exemptées de la participation au programme mais, de leur gré, elles peuvent choisir d'y prendre part. Les personnes qui offrent des soins à un membre de leur famille ayant des besoins particuliers, les bénéficiaires temporairement malades ou handicapés et les bénéficiaires en congé de maternité ou en congé parental sont temporairement exemptés du programme; les autres y travailleront jusqu'à 17 heures par semaine. En principe, le programme Ontario au travail devait être en place dans toute la province en 1998.

\section{La distinction entre les programmes d'employabilité et les programmes de travail obligatoire}

Il est important de distinguer les programmes de retour au travail ou d'employabilité et le travail obligatoire (workfare). Les programmes de retour au travail dépendent habituellement de l'inscription volontaire des participantes et des participants, offrent des programmes de recyclage ou de formation et soutiennent financièrement les personnes qui y participent.

Par contre, les programmes de travail obligatoire sont plus rigides et comportent des pénalités. Dans son sens le plus strict, le travail obligatoire exige que les bénéficiaires travaillent en échange de leur prestation d'aide sociale. Trois éléments clés définissent le travail obligatoire (Ontario Network of Employment Skills Training Projects/ONESTP 1995:1): c'est une activité de travail, 
la participation à celle-ci est obligatoire et il y a un échange direct entre la prestation d'une aide de dernier recours par l'État et l'accomplissement d'un travail forcé par l'assistée sociale.

\section{Les objectifs de la réforme ou... la carotte}

Le 12 juin 1997, la ministre des Services sociaux et communautaires de l'Ontario déposait le projet de loi 142, la Loi de 1997 sur la réforme de l'aide sociale (LRAS). Cette loi établit deux nouvelles lois : la Loi de 1997 sur le programme Ontario au travail (LPOT) et la Loi de 1997 sur le Programme ontarien de soutien aux personnes handicapées (LPOSPH). Comme on l'a mentionné plus tôt, cette réforme changera radicalement le visage et le fonctionnement de l'aide sociale en Ontario. La LPOT touchera les bénéficiaires du programme de l'aide sociale générale (ASG) et les chefs de famille monoparentale bénéficiaires des prestations familiales (PF). L'autre section de la réforme, la LPOSPH, s'appliquera aux personnes handicapées, y compris celles qui reçoivent actuellement des prestations familiales.

Les personnes politiques qui proposent la mise en oeuvre du programme de travail obligatoire plaident le bien-fondé d'une telle mesure en la liant à des objectifs ou à des concepts qui, de prime abord, semblent raisonnables. Les objectifs avoués du programme Ontario au travail, tels qu'indiqués dans le texte de loi, sont les suivants: reconnaitre la responsabilité individuelle et favoriser l'autonomie par l'emploi; fournir une aide financière provisoire à ceux qui sont le plus dans le besoin pendant qu'ils satisfont des obligations en vue de se faire employer et de le rester; servir efficacement les personnes qui ont besoin d'aide; comprendre l'obligation de rendre compte aux contribuables de l'Ontario.

Par ailleurs, dans les communications du ministère des Services sociaux et communautaires, on précise également que la réforme de l'aide sociale devrait poursuivre les objectifs suivants: placer les bénéficiaires dans des emplois permanents sans que le programme Ontario au travail n'enlève d'emploi rémunéré à qui que ce soit; permettre des économies aux contribuables en coupant les prestations aux personnes qui ne participent pas au programme 
(c'est-à-dire punir les personnes qui refusent de participer); couper dans les dépenses des programmes sociaux; réduire, voire éliminer, la fraude sociale; accroître les habiletés en emploi des participants et rehausser leur estime de soi.

\section{Les mots pour le dire}

"Les tenants du travail obligatoire estiment qu'il est également important d'employer un nouveau vocabulaire pour mieux comprendre la «justesse» de la réforme de l'aide sociale.»
Les tenants du travail obligatoire estiment qu'il est également important d'employer un nouveau vocabulaire pour mieux comprendre la «justesse» de la réforme de l'aide sociale. Dans un communiqué de presse émis le 12 juin 1997 et annonçant le dépôt du projet de loi 142 (LRAS), le ministère des Services sociaux et communautaires illustrait les modifications apportées à l'aide sociale en y insérant un petit lexique. Selon le Ministère, si les "prestataires» sont dorénavant des «participantes et participants», c'est parce que la "dépendance de la part des prestataires et de leurs enfants» sera remplacée par des «mesures leur permettant d'acquérir de l'estime de soi, de devenir autosuffisants et de briser le cycle de la dépendance»; c'est aussi parce qu'au lieu "d'inciter les gens à continuer à toucher l'aide sociale», on les incitera à retourner au travail; et c'est également parce que dorénavant, il sera «plus avantageux de travailler» que «de recevoir des prestations de l'aide sociale» (sic!).

Les «nouveaux» mots employés après la mise en oeuvre de la réforme possèdent un sens qui est tout sauf neutre. Ce qui devient clair, c'est que dorénavant, le gouvernement tiendra les prestataires de l'aide sociale (et leur famille) comme uniques responsables de leur situation. Ce vocabulaire inspire tout ce qu'il y a de contraire à la nouveauté puisqu'il perpétue de nombreux stéréotypes au sujet des prestataires de l'aide sociale. De plus, il contribue à confirmer leur rôle de boucs émissaires en leur faisant porter la responsabilité du marasme économique.

\section{Les revers du travail obligatoire ou... le bâton}

Dans la partie précédente, on peut constater que le gouvernement Harris, histoire de mieux nous faire avaler sa réforme de l'aide sociale, tend aux citoyennes et citoyens de l'Ontario une carotte 
«Il est toutefois

important de se souvenir que, tout comme la carotte que l'on brandit à l'âne pour le faire avancer, il $y$ a un bâton pas très loin derrière. Une fois la carotte consommée, il ne reste que le bâton pour faire bouger l'âne. Si la carotte (le discours) est un appât alléchant mais éphémère, le bâton nous ramène à la dure réalité qui, en contrepartie, subsiste et souvent fait mal.» qui semble plutôt alléchante. Les objectifs cités par la réforme et, à la rigueur, certains aspects du nouveau vocabulaire proposé pour enrober le tout, peuvent paraître à propos dans un contexte économique où «le déficit zéro» est devenu un mantra gouvernemental.

Il est toutefois important de se souvenir que, tout comme la carotte que l'on brandit devant l'âne pour le faire avancer, il y a un bâton pas très loin derrière. Une fois la carotte consommée, il ne reste que le bâton pour faire bouger l'âne. Si la carotte (le discours) est un appât alléchant mais éphémère, le bâton nous ramène à la dure réalité qui, en contrepartie, subsiste et souvent fait mal. Cette analogie n'a pas été employée à tout hasard car, après une étude approfondie de nombreuses expériences de travail obligatoire et une analyse de la loi 142 (LRAS), il devient plus qu'évident que les modalités d'application de cette réforme sont tout à fait contraires aux objectifs énoncés. Les personnes politiques savent toutefois enrober le tout en espérant que plusieurs mordront à l'appât «... comme on amuse un bébé avec un hochet... pour lui faire avaler sa purée de foie» (Pratte 1997:59).

Dans le texte qui suit, nous reprenons chacun des objectifs de la LRAS (c'est-à-dire «la carotte ou le discours») et nous offrons des renseignements qui permettront aux lectrices et aux lecteurs de mieux saisir quelques-uns des enjeux (c'est-à-dire «le bâton ou la réalité») qui se cachent sous les apparences.

\section{Selon le texte de loi, l'objectif 1, la carotte (ou le discours) c'est de... reconnaître la responsabilité individuelle et favoriser l'autonomie par l'emploi}

Et le bâton (ou la réalité), c'est que la reconnaissance de la responsabilité d'une personne présuppose qu'elle a la possibilité de faire un certain nombre de choix et qu'elle a accès à des ressources qui lui permettront de se prendre en main. Or, le travail obligatoire force les bénéficiaires à travailler un minimum d'heures par mois pour recevoir leurs prestations. De plus, ces personnes n'auront pas le choix du type de travail qu'elles devront effectuer ni de l'endroit où elles travailleront. 
"...la LRAS prévoit que le programme de l'aide sociale se transformera en système de prêt car, si une personne bénéficiaire vend sa maison, elle sera tenue de rembourser la totalité des prestations qu'elle a reçues avec les bénéfices de cette vente.»
Par ailleurs, la LRAS prévoit que le programme de l'aide sociale se transformera en système de prêt car, si une personne bénéficiaire vend sa maison, elle sera tenue de rembourser la totalité des prestations qu'elle a reçues avec les bénéfices de cette vente. Le gouvernement pourra aussi exiger, comme condition d'admissibilité à l'aide financière de base, «...qu'un bénéficiaire convienne de rembourser à l'administrateur l'aide qui sera fournie»" ${ }^{13}$ (Loi de 1997 sur la réforme de l'aide sociale: Partie I, article 13).

Enfin, la loi indique qu' «une fraction de l'aide financière de base peut être fournie directement à un tiers au nom du bénéficiaire si une somme est payable au tiers... à l'égard des coûts se rapportant aux besoins essentiels ou au logement..." (Loi de 1997 sur la réforme de l'aide sociale : Partie I, article 18). De plus, les décisions de verser de l'argent à un tiers, du montant versé et à qui cet argent sera versé ne pourront pas faire l'objet d'un appel. Le propriétaire d'un appartement ou un fournisseur de service n'auront-ils alors qu'à faire une demande de paiement direct à l'administration de l'aide sociale? Qu'arrivera-t-il si le coût du loyer est plus élevé que l'allocation prévue? Et qu'arrivera-t-il si une locataire veut retenir le loyer comme moyen de pression pour obliger un propriétaire à faire des réparations qu'il néglige ou refuse de faire?

Bref... la Loi de 1997 sur la réforme de l'aide sociale ne reconnaît aucunement la responsabilité individuelle, pas plus qu'elle ne favorise l'autonomie, puisqu'elle impose des contraintes extraordinaires sur les bénéficiaires et limite leurs choix.

\section{Selon le texte de loi, l'objectif 2, la carotte (ou le discours) c'est de... fournir une aide financière provisoire à ceux qui sont le plus dans le besoin pendant qu'ils satisfont des obligations en vue de se faire employer et le rester}

Et le bâton (ou la réalité), c'est qu'il est sous-entendu qu'il y a suffisamment d'emplois pour occuper toutes les personnes qui le veulent. Selon le ministère du Développement des ressources humaines du Canada, il y avait un peu moins de 31000 postes disponibles dans la région de Toronto en juin 1997. En mai 1997, Statistique Canada rapportait que 207000 personnes étaient 
"...le plus important problème que connaissent les pauvres ce n'est pas le manque d'éthique en emploi mais plutôt le manque d'éthique dans la rémunération et l'insuffisance d'emplois.» officiellement sans emploi dans la région de Toronto ${ }^{14}$. A ces données, ajoutons quelques chiffres de plus:

- $62 \%$ des nouveaux emplois créés dans la province de l'Ontario en 1997 sont à temps partiel;

- entre 1993 et 1996, le nombre d'emplois accessibles aux personnes ayant une scolarité inférieure à la $12^{\mathrm{e}}$ année a diminué de 100 000;

- la raison la plus souvent évoquée pour expliquer un retour à l'aide sociale est la perte d'emploi (dans $32 \%$ des cas);

- en Ontario, plus d'une personne sur dix, travaillant à temps plein toute l'année, gagnaient moins de 15000 \$ en 1995. Chez les femmes (travaillant à temps plein toute l'année), $14 \%$ ont un revenu inférieur à 15000 \$ et $28 \%$ gagnaient moins de 20000 \$ (le taux de pauvreté pour un ménage de deux personnes).

Bref... peut-être que le plus important problème que connaissent les pauvres, ce n'est pas le manque d'éthique en emploi mais plutôt le manque d'éthique dans la rémunération et l'insuffisance d'emplois.

\section{Selon le texte de loi, l'objectif 3, la carotte (ou le discours) c'est de... servir efficacement les personnes qui ont besoin d'aide}

Et le bâton (ou la réalité), c'est que le gouvernement Harris prétend qu'avant sa réforme, il était plus avantageux de recevoir des prestations de l'aide sociale que de travailler. C'est malheureusement aussi l'opinion d'une partie de la population ontarienne. Or, les chiffres réels dépeignent un portrait tout autre de la situation.Voici un aperçu sommaire des montants que peuvent toucher deux types de bénéficiaires de l'aide sociale en Ontario (MSSC 1997b) : 
Tableau 1 - Montant maximum de prestations de l'aide sociale accordé selon le type de bénéficiaire et selon le programme comparé au seuil de faible revenu

\begin{tabular}{|c|c|c|c|c|c|}
\hline \multirow[t]{2}{*}{ Type de bénéficiaire } & \multicolumn{2}{|c|}{$\begin{array}{l}\text { Prestations d'aide } \\
\text { sociale générale }\end{array}$} & \multicolumn{2}{|c|}{$\begin{array}{l}\text { Prestations } \\
\text { familiales }\end{array}$} & \multirow{2}{*}{$\begin{array}{c}\text { Seuil de } \\
\text { pauvreté }\end{array}$} \\
\hline & Mensuelles & Annuelles & Mensuelles & Annuelles & \\
\hline Adulte vivant seul-e & $520 \$$ & $6240 \$$ & $930 \$$ & $11160 \$$ & $16061 \$$ \\
\hline $\begin{array}{l}\text { Adulte avec } 2 \text { enfants } \\
\text { à charge }\end{array}$ & $1205 \$$ & $14460 \$$ & $1184 \$$ & $14208 \$$ & $27672 \$$ \\
\hline
\end{tabular}

Source :

Le Conseil national de bien-être social, Profil de la pauvreté, 1995, publié en 1997, selon le seuil de faible revenu de Statistique Canada, base de 1986 pour 1996.

Par ailleurs, si on étudie les possibilités de revenu selon les normes minimales d'emploi de l'Ontario, on peut facilement constater qu'il est certes beaucoup plus avantageux d'empocher un revenu d'emploi que des prestations de l'aide sociale. Prenons l'exemple d'une personne occupant un emploi à temps plein dans le secteur de la vente au détail et qui toucherait le salaire minimum en vigueur en Ontario (en janvier 1998), soit 6,85 \$ de l'heure. Ce type de personne travaille généralement 44 heures par semaine. Elle peut donc toucher un revenu mensuel de 1306 \$ (revenu annuel de 15672 \$). Or, en 1990, une personne seule apte au travail recevait en aide sociale l'équivalent de $61 \%$ du salaire minimum provincial. En 1996, elle ne recevait plus que $44 \%$ (Conseil national du bien-être social 1997:73).

Dans un autre ordre d'idées, peu de temps après l'annonce d'une réduction de $21,6 \%$ des prestations de l'aide sociale en octobre 1995, Dave Tsubouchi, alors ministre des Services sociaux et communautaires, diffusait une liste d'épicerie. Selon cette liste, une personne vivant seule pouvait se nourrir convenablement pour la modique somme de $90 \$$ par mois. Cette liste a fait couler beaucoup d'encre et pour cause. Les pâtes alimentaires, le pain, la saucisse de Bologne (le bon vieux «baloney») et le thon (vraiment pas cher) étaient les articles principaux qui s'y trouvaient. Cette 
précieuse liste ne contenait pratiquement pas de légumes, aucun fruit ni sauce d'accompagnement aux pâtes alimentaires.

Bref... la réforme du gouvernement Harris ne sert pas efficacement les personnes qui ont besoin d'aide. Si les bénéficiaires de l'aide sociale n'arrivent pas à subvenir à leurs besoins essentiels, ce n'est pas parce qu'ils manquent de connaissances en planification budgétaire ni à cause de folles dépenses, mais bien parce qu'ils ont un revenu nettement inférieur au seuil de pauvreté.

Selon le texte de loi, l'objectif 4, la carotte (ou le discours) c'est de... comprendre l'obligation de rendre compte aux contribuables de l'Ontario

Et le bâton (ou la réalité), c'est que le ministère des Services sociaux et communautaires se fait un devoir de publier tous les mois le nombre de cas d'aide sociale et de préciser s'il s'agit d'une augmentation ou d'une diminution par rapport au mois précédent. De plus, ce Ministère publie régulièrement le nombre d'appels qu'il reçoit sur sa ligne téléphonique anti-fraude.

Toutefois, il est impossible de trouver des chiffres (avec preuve à l'appui) démontrant: le nombre de bénéficiaires ayant quitté l'aide sociale pour un emploi; combien de ces ex-bénéficiaires occupent un emploi permanent ou temporaire; le salaire que reçoivent maintenant ces personnes; le coût de l'administration du programme Ontario au travail ${ }^{15}$; le coût de «l'escouade antifraude» du gouvernement Harris. Bref... le gouvernement Harris ne fait que rendre des comptes partiels au public. Il est essentiel de tenir compte de l'ensemble des renseignements précités afin de mesurer si la réforme actuelle en vaut la chandelle.

\section{Selon des communications du Ministère, l'objectif 5, la carotte (ou le discours) c'est de... placer les bénéficiaires dans des emplois permanents sans que le programme Ontario au travail n'enlève aucun emploi rémunéré à qui que ce soit}

Et le bâton (ou la réalité), c'est que la région de Muskoka annonçait, entre autres, les postes suivants destinés aux participantes et participants du programme Ontario au travail (Workfare Watch 
"...plusieurs emplois qui, en d'autres temps auraient été rémunérés, seront comblés par des bénéficiaires qui toucheront leurs prestations d'aide sociale en échange.» 1997b : 5): tuteur/tutrice en alphabétisation et agent-e de bureau, agent-e de bureau à un collège, aide en garderie, assistant-e au centre de ressources. D'autres emplois qui seront comblés par des bénéficiaires de l'aide sociale en retour de leurs prestations incluent: aide à l'information dans une bibliothèque scolaire, peintre/ jardinier, aide bibliothécaire, chercheur-e affecté aux archives et agent-e de bureau à Bracebridge. Et, dans le comté d'Algoma, 147 bénéficiaires de l'aide sociale sont assignés aux tâches suivantes: peinture des bureaux municipaux, travail clérical et installation de rampes d'accès pour les fauteuils roulants. Enfin, le comté de Halton prévoit ouvrir 360 placements de travail obligatoire et identifie les garderies et les foyers pour personnes âgées parmi les plus importants centres de placements potentiels (Workfare Watch 1997a:1).

Bref... comme on peut le constater, les municipalités, les conseils scolaires et les organismes communautaires réaliseront d'importantes économies puisque plusieurs emplois qui, en d'autres temps auraient été rémunérés, seront comblés par des bénéficiaires qui toucheront leurs prestations d'aide sociale en échange. De plus, ce programme ne place pas les bénéficiaires dans des emplois permanents.

\section{Selon des communications du Ministère, l'objectif 6, la carotte (ou le discours) c'est de... permettre des économies aux contribuables en coupant les prestations aux personnes qui, de mauvaise foi, refusent de participer au programme}

Et le bâton (ou la réalité), c'est qu'un bénéficiaire a été avisé un jour à l'avance qu'il devait participer à une «session d'orientation» d'une durée de deux jours. La première journée portait sur la santé et la sécurité au travail et la seconde traitait des habiletés en entrevue et de la préparation d'un curriculum vitae. L'homme qui avait été convoqué ne s'est pas rendu à la session parce qu'il avait des engagements préalables et parce qu'il avait récemment suivi une formation semblable. On a immédiatement coupé ses prestations pour trois mois. Bien que le bénéficiaire ait interjeté appel devant la Commission de révision de l'aide sociale et qu'il 
ait obtenu gain de cause (Workfare Watch Bulletin 1997:2), cet exemple illustre le risque d'abus de la part du gouvernement et ce, au détriment des droits fondamentaux des assistés sociaux.

Bref... le programme Ontario au travail permet effectivement au gouvernement de faire, à court terme, des économies. Cependant, non seulement punit-on les personnes qui sont récalcitrantes, mais ce, à partir de motifs déraisonnables.

\section{Selon des communications du Ministère, l'objectif 7, la carotte (ou le discours) c'est de... couper dans les dépenses des programmes sociaux}

Et le bâton (ou la réalité), c'est que rien ne permet au gouvernement actuel d'affirmer que les mesures entreprises jusqu'à maintenant ont entraîné une diminution des dépenses des programmes. L'expérience américaine démontre d'ailleurs que les programmes de travail obligatoire sont, au contraire, très onéreux. La diminution des prestations de l'aide sociale n'est qu'un des facteurs dont on doit tenir compte dans le calcul de tels chiffres. On ne saura évaluer s'il y a eu de réelles économies que lorsqu'on connaitra l'impact de la réforme proposée sur d'autres dépenses publiques telles que le logement social, les services de santé, les services policiers, le système carcéral, etc.

\section{Selon des communications du Ministère, l'objectif 8, la carotte (ou le discours) c'est de... réduire, voire éliminer la fraude sociale}

Et le bâton (ou la réalité), c'est que lorsque le gouvernement emploie le mot "fraude», il entend non seulement les cas de délit

"...la plupart des paiements fautifs ne sont pas le résultat de tentatives délibérées de fraude.» criminel mais également tous les paiements fautifs. Cependant, la plupart des paiements fautifs ne sont pas le résultat de tentatives délibérées de fraude. On peut qualifier de paiement fautif les cas où il n'y a pas eu de vérification des conditions d'admissibilité, ceux où une personne dans le besoin reçoit un montant d'argent supplémentaire auquel elle n'a pas droit pour des raisons techniques et même ceux qui surviennent lorsqu'une erreur a été commise au sujet des conditions d'admissibilité d'une personne (autant de la part de la ou du bénéficiaire que de l'administration de l'aide sociale). 
«En mars 1997, 92 allégations de fraude ont été référées aux services policiers pour enquête, 32 l'ont été au Procureur de la couronne et il y a eu un grand total de 9 condamnations ${ }^{16} \cdot A u$ cours de cette période, plus de 600000 personnes (célibataires ou chefs de famille) recevaient des prestations de l'aide sociale."
Peu de temps après son élection, le gouvernement Harris annonçait la mise en place de sa ligne téléphonique anti-fraude. Depuis, quelque 18000 appels ont été logés à cette ligne (MSSC 1997c). En mars 1997, 92 allégations de fraude ont été référées aux services policiers pour enquête, 32 l'ont été au Procureur de la couronne et il y a eu un grand total de 9 condamnations ${ }^{16}$. Au cours de cette période, plus de 600000 personnes (célibataires ou chefs de famille) recevaient des prestations de l'aide sociale.Y a-t-il vraiment lieu de croire que la fraude criminelle est largement répandue dans les rangs de l'aide sociale?

Si elle est adoptée par l'Assemblée législative, la LRAS reconnaitra des pouvoirs policiers sans précédent aux gestionnaires de l'aide sociale. La nouvelle loi confere à la province et aux municipalités le pouvoir d'établir des unités de répression des fraudes et autorise la nomination d'agents d'aide au recouvrement qui auront le pouvoir de demander un mandat de perquisition et d'agir en vertu de celui-ci ${ }^{17}$.Y a-t-il raison de croire que l'Ontario se dirige vers la mise en place d'un État policier?

\section{Selon des communications du Ministère, l'objectif 9, la carotte (ou le discours) c'est d'accroître les habiletés en emploi des participants et rehausser leur estime de soi}

Et le bâton (ou la réalité), c'est que les personnes contraintes à participer aux différents programmes de travail obligatoire au Canada et en Ontario sont surtout affectées à des tâches d'entretien et des travaux manuels. En quoi ce type «d'expérience de travail» permettra-t-il aux bénéficiaires de se placer en emploi (de façon permanente), à une époque où l'informatique et la robotique règnent dans le monde des affaires?

Comment peut-on rehausser son estime de soi alors qu'on est pointé du doigt, culpabilisé pour sa situation économique, stigmatisé par la classe moyenne qui en a assez de payer toujours plus d'impôts et de taxes? Comment peut-on cultiver une meilleure estime de soi alors que tout un chacun peut connaitre notre situation de vulnérabilité économique et alors qu'on ne bénéficie pas d'une reconnaissance sociale parce qu'il nous est 
«...depuis les expériences canadiennes et ontariennes des années 30 , il est clair que les programmes de travail obligatoire ne permettent pas aux participants d'accroitre leurs habiletés en emploi ni de rehausser leur estime de soi." impossible de contribuer au développement de notre communauté, alors qu'aucun débouché d'emploi ne s'offre à nous?

Bref... depuis les expériences canadiennes et ontariennes des années 30, il est clair que les programmes de travail obligatoire ne permettent pas aux participants d'accroître leurs habiletés en emploi ni de rehausser leur estime de soi. En contrepartie, ces programmes permettent à l'État de projeter une image de main d'oeuvre à bon marché; une image qui, à tout coup, sait plaire au monde des affaires.

\section{Le cas des chefs de famille monoparentale}

Comme critère d'admissibilité à l'aide sociale, la réforme stipule que les femmes chefs de famille monoparentale (c'est-à-dire plus de 200000 femmes en Ontario) devront, entre autres, accepter un placement de travail obligatoire. Quoique la ministre Janet Ecker ait affirmé que ce critère s'appliquait uniquement aux chefs de famille monoparentale dont les enfants étaient d'âge scolaire, la loi ne mentionne pas d'exemption à cet effet.

De plus, même s'il impose une corvée de travail obligatoire aux femmes chefs de famille monoparentale, le gouvernement n'est pas tenu légalement d'assurer l'accès à des services de garde de qualité et à prix abordable. Déjà, l'Ontario connaît une importante pénurie de services de garde subventionnés et les listes d'attente abondent. La liste d'attente pour des services de garde subventionnés dans la municipalité métropolitaine de Toronto compte quelque 18000 enfants (y inclus 8500 enfants bénéficiaires de l'aide sociale). Pourtant, le programme Ontario au travail (région de Toronto) n'a prévu de créer que 2500 nouvelles places en garderie. Le Metro Toronto Community Services Department a estimé qu'au moins 42500 enfants auront besoin de services de garde lorsque les 20300 familles bénéficiaires de l'aide sociale seront tenues d'effectuer des travaux obligatoires à compter de janvier $1998^{18}$. 


\section{Que savons-nous de la monoparentalité?}

Les femmes chefs de famille monoparentale et leurs enfants font partie des groupes les plus vulnérables de la société et sont surreprésentées aux rangs des pauvres. Selon les statistiques de 1994, 74 p.100 des femmes chefs de famille monoparentale étaient bénéficiaires de l'aide sociale (revenu annuel moyen de $9694 \$$ ), $43 \%$ touchaient un salaire (revenu annuel moyen de 6829 \$) et seulement $20 \%$ recevaient un appui financier du conjoint (revenu annuel moyen de 3254 \$) (Conseil national du bien-être social 1996). La même année, 57,3 \% des Canadiennes chefs de famille monoparentale vivaient sous le seuil de pauvreté (Conseil national du bien-être social 1996:7).

\section{Et les enfants dans tout ça?}

"Le gouvernement prétend que trop de bénéficiaires, $y$ compris les femmes chefs de famille monoparentale, ont une insuffisance de compétences en emploi et ne possèdent pas

suffisamment d'habitudes (d'éthique) d'emploi.»
On compte plus de 400000 enfants (ou $40 \%$ des bénéficiaires) aux rangs de l'aide sociale en Ontario et $93 \%$ d'entre eux sont nés avant que leurs parents ne soient bénéficiaires (Stapleton 1994).

Le gouvernement prétend que trop de bénéficiaires, y compris les femmes chefs de famille monoparentale, ont une insuffisance de compétences en emploi et ne possèdent pas suffisamment d'habitudes (d'éthique) d'emploi. Les «Mikey Macouttes» vont jusqu'à insinuer, à mots couverts, que les bénéficiaires sont des personnes paresseuses et fainéantes qui préferent vivre aux crochets de la société plutôt que de s'en sortir et que certaines femmes donnent naissance à des enfants pour soutirer de l'argent du gouvernement.Ainsi, par ce discours, il parait justifié, à leurs yeux, d'appliquer des mesures de travail obligatoire et de limiter l'accès aux prestations de l'aide sociale pour «inciter les bénéficiaires à retourner au travail». Il est plus que temps de faire éclater ce mythe de la paresse et de l'abus.Voici quelques chiffres au sujet de la réalité des femmes chefs de famille monoparentale. En 1990, 
«Presque $48 \%$ des femmes chefs de famille monoparentale faisant partie de la population active étaient en chômage à cause d'une mise à pied ou de l'élimination de leur poste.» alors que $75 \%$ des femmes chefs de famille monoparentale avaient un revenu d'emploi, $52 \%$ de ces familles vivaient sous le seuil de pauvreté (Statistique Canada 1992: 37). Le taux de chômage chez les femmes chefs de famille monoparentale s'élevait à 16,8\% en 1991 alors que $10 \%$ de l'ensemble de la population de l'Ontario était au chômage. Presque $48 \%$ des femmes chefs de famille monoparentale faisant partie de la population active étaient en chômage à cause d'une mise à pied ou de l'élimination de leur poste.

Les femmes ont été particulièrement touchées par les récentes pertes d'emplois dans les secteurs public et communautaire. Une des répercussions de ces pertes d'emplois, c'est qu'un plus grand nombre de femmes occupent des emplois à temps partiel. En 1991,71\% des travailleurs à temps partiel étaient des femmes. En 1992, il est estimé que les femmes ont perdu 125000 emplois à temps plein pour en regagner 69000 à temps partiel ${ }^{19}$.

\section{En guise de conclusion... Le syndrome de Pinocchio est bien vivant en Ontario}

Pour diminuer le nombre de personnes dépendant de l'aide sociale et redresser la situation des finances publiques, le gouvernement Harris a dit, en campagne électorale, qu'il s'engageait à «investir 500 millions de dollars dans des programmes nouveaux et innovateurs pour aider les plus démunis» (Parti progressiste conservateur de l'Ontario 1995: 9). Lorsque le gouvernement Harris a déposé, en 1997, le projet de loi 142, il a affirmé que cette réforme de l'aide sociale «aurait pour but l'autonomie». Par contre, ce que la réforme de l'aide sociale dicte, c'est, entre autres, d'imposer des travaux forcés aux bénéficiaires de l'aide sociale et d'exiger que ces personnes remboursent l'État pour l'aide qu'elles auront reçue.

En comparant le discours à la réalité, il nous parait évident que les arguments pour justifier l'introduction d'un programme de travail obligatoire en Ontario, et par extension, les nombreuses 
contraintes qu'impose la réforme de l'aide sociale aux personnes les plus démunies de notre société, sont cousus de mensonges et déforment la réalité.

Il est maintenant connu et reconnu de tous que les politiciens mentent de façon éhontée à la population. En des termes plus polis, on appelle souvent ces mensonges de la démagogie. Les tenants de politiques néolibérales sont passés maîtres dans l'art de pratiquer le mensonge ou la démagogie: ils savent très bien exploiter les émotions, les passions, l'ignorance et les préjugés pour obtenir et conserver le pouvoir (Paradis 1997: 53).

"L'écart grandissant entre les classes sociales et l'accroissement de la pauvreté nous interrogent dans notre rôle d'intervenantes et d'intervenants.»
L'écart grandissant entre les classes sociales et l'accroissement de la pauvreté nous interrogent dans notre rôle d'intervenantes et d'intervenants. Le système de l'aide sociale doit être réformé pour répondre aux impératifs de ce tournant de siècle, nous en convenons. Cette réforme cependant, ne doit pas se faire à n'importe quel prix et encore moins, sous le couvert du mensonge.

Selon Pratte (1997:84), «la vitalité d'une démocratie dépend de la qualité du débat électoral». Il poursuit en ajoutant que «pour qu'il y ait démocratie, il ne suffit pas qu'il y ait des élections. Il faut aussi que celles-ci soient des moments de vérité, au sens littéral de l'expression. Ceux qui aspirent à gouverner doivent se présenter et exposer leurs idées avec franchise et honnêteté. Sans quoi la démocratie est faussée». Mike Harris, le Premier ministre de l'Ontario, a lui-même dit que «les politiciens ont peur de faire face aux questions difficiles, ou même d'en parler. Ils ne s'en tirent que trop bien grâce au statu quo» (Pratte 1997:112).Auronsnous le courage de mettre fin au statu quo?

\section{Bibliographie}

CHOLETTE, C. (1997). Inventaire des politiques néolibérales adoptées par le gouvernement de l'Ontario au cours de la méga-semaine et de certaines recommandations de la Commission de révision des formalités administratives, Ottawa, Table féministe francophone de concertation provinciale de l'Ontario. 
CONSEIL NATIONAL DU BIEN-ÊTRE SOCIAL (1997). Un autre regard sur la réforme de l'aide sociale, Ottawa, Ministre des Travaux publics et services gouvernementaux Canada.

CONSEIL NATIONAL DU BIEN-ÊTRE SOCIAL (1996). Poverty Profile 1994, Ottawa, printemps. CONSEIL NATIONAL DU BIEN-ÊTRE SOCIAL (1995). Social Security Backgrounder \# 4:Working for Welfare, Ottawa.

CÔTÉ, Andrée (1997). «Quelques notes à propos des plus récentes politiques du gouvernement Harris», dans C. Cholette et A. Côté, éd. L'impact des politiques néolibérales sur les droits des femmes, Compte rendu du forum tenu le 22 mars 1997 à Toronto, Ottawa, Table féministe francophone de concertation provinciale de l'Ontario et Centre de recherches féministes de l'universitéYork, 19-31.

ECKER,J. (1997). Notes pour l'allocution de Mme Janet Ecker, ministre des Services sociaux et communautaires, lors de la présentation en deuxième lecture de la Loi sur la réforme de l'aide sociale, Toronto, 19 août.

HARDINA, Donna (1997). "Workfare in the U.S. Empirically-Tested Programs or Ideological Quagmire?», dans Eric Shragge, éd. Workfare: Ideology for a New Under-Class, Toronto, Garamond Press, Toronto, 131-148.

HERNSTEIN, Richard J. et Charles MURRAY (1994). The Bell Curve: Intelligence and Class Structure in American Life, New York, The Free Press.

KIRP, David L. (1986). «The California Work/Welfare Scheme», The Public Interest, $\mathrm{n}^{\circ}$ 83, printemps. MEAD, Lawrence (1984). Beyond Entitlement, New York, The Free Press.

MINISTÈRE DES SERVICES SOCIAUX ET COMMUNAUTAIRES DE L'ONTARIO (s.d.). Points saillants du programme Ontario au travail, fiche de renseignements, Toronto.

MINISTÈRE DES SERVICES SOCIAUX ET COMMUNAUTAIRES DE L'ONTARIO (1997a). L'Ontario tient sa promesse sur la réforme de l'aide sociale, communiqué de presse émis le 12 juin, Toronto.

MINISTÈRE DES SERVICES SOCIAUX ET COMMUNAUTAIRES DE L'ONTARIO (1997b). Social Assistance and Pension Rate Table, Statistical and Analysis Unit, Social Assistance Programs Branch, révisé le $1^{\text {er }}$ avril.

MINISTÈRE DES SERVICES SOCIAUX ET COMMUNAUTAIRES (1997c). Résultats obtenus grâce à la ligne téléphonique anti-fraude du gouvernement, communiqué de presse émis le 9 avril, Toronto.

MINISTÈRE DES SERVICES SOCIAUX ET COMMUNAUTAIRES (1998). Le nombre de bénéficiaires de l'aide sociale en décembre rendu public, communiqué de presse émis le 9 janvier.

MITCHISON, R. (1991). Coping with Destitution: Poverty and Relief in Western Europe, Toronto, University of Toronto Press.

MULLALY, Robert (1997). «The Politics of Workfare: NB Works», dans Eric Shragge (dir. de pub.), Workfare: Ideology for a New Under-Class, Toronto, Garamond Press, Toronto, 3557.

MURRAY, Charles (1984). Losing Ground, New York, Basic Books.

ONTARIO NETWORK OF EMPLOYMENT SKILLS TRAINING PROJETS (1995). Singing for our Supper: A Review of Workfare Programs, Toronto, juillet.

ONTARIO SOCIAL SAFETY NETWORK (23 août, 1997a). Welfare Reform \& the Labour Market: Are There Enough Jobs?, Toronto, document d'information.

ONTARIO SOCIAL SAFETY NETWORK (23 août, 1997b). Welfare Reform \& Welfare Fraud The Real Issues, Toronto, document d'information. 
ONTARIO SOCIAL SAFETY NETWORK (23 août, 1997c). Welfare Reform E Single Mothers, Toronto, document d'information.

PARADIS, Blanche (1997). «Les politiques néolibérales et leur impact sur les groupes communautaires ou le syndrome de Pinocchio», dans Chantal Cholette et Andrée Côté, éd. L'impact des politiques néolibérales sur les droits des femmes, Ottawa, Table féministe francophone de concertation provinciale de l'Ontario, 53-57.

PRATTE, André (1997). Le syndrome de Pinocchio, Montréal, Les Éditions du Boréal.

SHRAGGE, Eric et Marc-André DENIGER (1997). "Workfare in Quebec», dans Eric Shragge (éd.) Workfare: Ideology for a New Under-Class, Toronto, Garamond Press, 59-83.

STAPLETON,J. (1994). Report on Social Assistance Programs in Ontario, Toronto, ministère des Services sociaux et communautaires.

STATISTIQUE CANADA (1992). Lone Parent Families in Canada, Ottawa, Target Groups Project.

STRUTHERS, J. (1996). Can Workfare Work? Reflections From History, Ottawa, The Caledon Institute of Social Policy.

STRUTHERS, J. (1993). No Fault of Their Own: Unemployment and the Canadian Welfare State 1914 1941, Toronto, University of Toronto Press.

Workfare Watch (1997a). vol. 1, no 4, mars.

Workfare Watch (1997b). vol. 1, no 5, juin.

Workfare Watch Bulletin (1997). vol. 1, no 4, juin.

\section{Notes}

1. Cet article a été rédigé à partir d'un document de réflexion préparé par le groupe d'étude sur le travail obligatoire. Initié par la Table féministe francophone de concertation provinciale de l'Ontario, ce groupe s'est donné pour mandat de colliger les renseignements essentiels au sujet du programme de travail obligatoire Ontario au travail et de les présenter, sous différents formats (dont une vidéocassette), aux communautés de l'Ontario français. Cette campagne d'information sera suivie d'une série de forums communautaires régionaux (à Ottawa, Toronto, Sudbury et Timmins) au cours du printemps 1998.

2. Je tiens à remercier Andrée Côté, Jacinthe Michaud et Lise Tardif, mes collègues du groupe d'étude, qui ont commenté l'ébauche de ce texte. Je témoigne aussi toute ma reconnaissance à Geneviève Guindon, Blanche Paradis et particulièrement à Lyne Bouchard, mes complices de travail, qui ont généreusement injecté idées et sang-froid aux moments les plus opportuns de la rédaction. 
3. Le gouvernement Harris a annoncé une foule d'importantes réformes et déposé plusieurs projets de loi au cours de la semaine du 13 janvier 1997. Pour obtenir de plus amples renseignements au sujet de ces réformes, consulter C. Cholette (1997).

4. Cette commission, présidée par le député conservateur Frank Sheehan, déposait son rapport définitif le 21 janvier 1997.

5. Les données historiques proviennent principalement de J. Struthers, 1996.

6. Traduction libre de "All whom God, not their idleness, makes poor, must be relieved", cité dans Mitchison, R. 1991:21.

7. Traduction libre de «Relief for Workers, Nothing for Shirkers».

8. Il n'est d'ailleurs pas surprenant que la dépression prenne fin en 1941 soit, deux ans après le déclenchement de la deuxième guerre mondiale. L'histoire nous démontre que «'industrie de la guerre» permet aux systèmes capitalistes de régler leurs problèmes économiques.

9. La loi prévoyait, entre autres, que le fédéral partagerait à nouveau les coûts de l'aide sociale avec les provinces permettant ainsi d'offrir des prestations d'aide sociale aux personnes aptes au travail qui auraient épuisé leurs prestations d'assurancechômage ou qui n'y seraient pas éligibles.

10. Le «Canada Assistance Plan (CAP)» en anglais.

11. "Canada Health and Social Transfen" en anglais.

12. Le texte original de ce document contenait une analyse des programmes similaires de travail obligatoire aux États-Unis, au Québec et au Nouveau-Brunswick. Le format de publication ne permettait malheureusement pas d'inclure la critique de ces expériences.

13. Une personne bénéficiaire sera tenue non seulement de travailler bénévolement dans sa communauté pour toucher son chèque d'aide sociale, mais aussi de rembourser l'argent reçu lorsqu'elle décrochera un emploi rémunéré.

14. Les personnes officiellement sans emploi sont celles qui étaient en chômage et cherchaient activement à se placer en emploi. Ce chiffre ne compte pas les personnes qui ont cessé de chercher un emploi parce qu'elles étaient découragées ni les 
personnes occupant un emploi à temps partiel qui pourraient chercher un emploi à temps plein. Les chefs de famille monoparentale ne cherchant pas activement un emploi sont aussi exclues de ce chiffre. Cependant, selon la LRAS, elles seront considérées comme des personnes aptes au travail et devront chercher un emploi (Ontario Social Safety NetWork 1997a:3).

15. Selon un fonctionnaire, l'institution d'un Programme de travail obligatoire complet en Ontario pourrait coûter jusqu'à un milliard de dollars (Conseil national du bien-être social 1997:69).

16. Seulement 6,8 p. 100 des appels ont débouché sur une action quelconque (couper ou réduire des prestations) (Ontario Social Safety NetWork 1997b:1).

17. La loi permet également au gouvernement - et c'est ce qui est le plus inquiétant - d'accorder des pouvoirs policiers additionnels à ces agents d'aide au recouvrement par voie de règlement.

18. Ce chiffre ne comprend pas les besoins des chefs de famille de la région de Toronto recevant présentement des prestations familiales qui seront aussi tenus de participer au programme Ontario au travail.

19. Armstrong, 1991, p.32, cité par le Ontario Social Safety NetWork 1997c: 3. 